\title{
A POLÍTICA DE IMPLEMENTAÇÃO DO PLANO NACIONAL DE SAÚDE A NÍVEL LOCAL EM PORTUGAL
}

\author{
Denise Capela dos Santos
}

ORCID ID:0000-0002-5794-5203;

CIÊNCIA ID: 0B10-1C58-71EB,

dsantos@autonoma.pt

O Plano Nacional de Saúde (PNS) fixa objetivos prioritários de ganhos em saúde na população e as respetivas diretrizes de ação, para alcance de determinadas metas nacionais de saúde, definidas pelo Governo Português, com base nas orientações provenientes do Gabinete Geral para a Europa da Organização Mundial de Saúde.

Este Plano deverá ser a base de elaboração dos Planos Regionais de Saúde (PRS) e dos Planos Locais de Saúde (PLS), de forma a garantir a implementação e cumprimento das metas referidas, em todo o território do país.

De facto, agregados os objetivos e metas locais, definidas em cada Plano Local de Saúde, considerando as especificidades de cada local, deverão estar reunidas as condições para alcance das metas e objetivos dos respetivos Planos Regionais de Saúde. Do mesmo modo, reunidos os objetivos e metas dos Planos Regionais de Saúde, considerando as particularidades de cada região, deverão estar salvaguardadas as metas e objetivos nacionais que constam no Plano Nacional de Saúde.

\section{O PLANO NACIONAL DE SAÚDE}

Nos últimos cinco anos, Portugal teve três Planos Nacionais de Saúde: o PNS 2011-2016, abandonado devido à crise económica e financeira que o país começou a atravessar de forma mais evidente em 2009; o PNS 2012-2016, elaborado entretanto, por adaptação do anterior aos recursos de que o país dispunha; e o PNS 2012-2016, com extensão a 2020 (PNS 2012-20162020), apresentado em 2014 em Portugal, para alinhamento do país com a política europeia "Saúde 2020", construída pelo Gabinete para a Europa da Organização Mundial de Saúde (OMS), em 2012.

Este PNS pretende dar uma resposta ao cenário epidemiológico e demográfico do país, contribuindo para que se incentive a natalidade, para que os jovens venham a ser adultos mais saudáveis e para que existam respostas rápidas e eficazes aos desafios lançados tanto pelas doenças de evolução prolongada, como por episódios agudos de doença (DGS, 2015)b.

O PNS 2012-2016-2020 quatro eixos estratégicos que transitaram do PNS anterior: Cidadania em Saúde, Equidade e Acesso Adequado aos Cuidados de Saúde, Qualidade na Saúde e Políticas Saudáveis (DGS, 2015)a , enquadrados na estratégia da "Saúde 2020", de melhoria da 
Working Paper

Departamento de Ciências Económicas e Empresariais

Universidade Autónoma de Lisboa

saúde de todos e da redução de iniquidades em saúde, com uma governação mais participativa e maior liderança para a saúde (WHO, 2012).

Para alcançar a estratégia definida, o PNS 2012-2016-2020, define três grandes metas: 1) a redução para menos de $20 \%$ da taxa de mortalidade prematura (abaixo dos 70 anos), 2) o aumento em $30 \%$ da esperança de vida saudável aos 65 anos de idade e 3) a redução dos fatores de risco relacionados com as doenças não transmissíveis, nomeadamente o consumo e exposição ao fumo do tabaco e a obesidade infantil (DGS, 2015)a , embora existam condicionantes ao desenvolvimento atempado do sistema de informação (DGS, 2015) ${ }^{\text {b }}$ para o seu alcance.

A "Saúde 2020" apresenta quatro áreas prioritárias: 1) dar prioridade à saúde ao longo de toda a vida, conferindo poder sobre a saúde aos cidadãos, 2) combater as doenças com maior impacto na Europa, 3) melhorar a capacidade de resposta da saúde pública e centralizar o sistema nas pessoas, e 4) criar ambientes de suporte e comunidades resilientes (WHO 2012).

Temos portanto um PNS atual alinhado com a política para a Europa 2020, conduzido por princípios e valores bem definidos, com foco nos ganhos em saúde da população.

O Plano Nacional de Saúde é implementado através de programas, projetos, atividades e ações operacionalizadas por diferentes agentes e dirigidas a necessidades específicas da população Portuguesa, a nível nacional, regional e local.

\section{OS PLANOS REGIONAIS DE SAÚDE}

O documento de Resenha dos Planos de Saúde (DGS, 2015) , integra os perfis e planos locais e regionais de saúde existentes, por ACES/ ULS e por ARS, respetivamente, para Portugal Continental. Este documento indica que os Planos Regionais de Saúde do Alentejo e de Lisboa e Vale do Tejo se encontram em fase de elaboração.

No entanto, realizada uma pesquisa online sobre o tema, pelo Google, usando a palavra "Plano regional de saúde", encontraram-se os seguintes planos:

1. Plano Regional de Saúde do Centro 2015-2016, da ARS do Centro (2015);

2. Plano Regional de Saúde 2013-2016, da ARS de LVT (2013);

3. Plano Regional de Saúde do Norte 2014-2016, da ARS do Norte (2014);

4. Plano Regional de Saúde 2014-2016, da Secretaria Regional de Saúde da Região Autónoma dos Açores (2014);

5. Plano Estratégico do Sistema Regional de Saúde: 2011-2016, da Secretaria Regional dos Assuntos Sociais da Região Autónoma da Madeira (2011)

Não se encontrou o Plano Regional de Saúde da ARS do Algarve, mesmo após pesquisa no respetivo site. O site da ARS do Alentejo informa que o Plano Regional de Saúde desta ARS está em fase de discussão.

Encontraram-se, no entanto, os Planos Regionais das Regiões Autónomas, que não constavam no documento da DGS e que seriam importantes integrar para avaliação da coerência de 
Working Paper

Departamento de Ciências Económicas e Empresariais

Universidade Autónoma de Lisboa

procedimentos e de planos de ação por região, para alcance de objetivos nacionais e implementação do Plano Nacional de Saúde em todo o território nacional.

Por avaliação dos Planos Regionais disponíveis na internet e considerando que, em 2010, se planeava um Plano Nacional de Saúde 2011-2016, verifica-se que a Região Autónoma da Madeira foi a única região que definiu um Plano Regional de Saúde, respeitando o intervalo de tempo do Plano Nacional, de 2011 a 2016. O Plano Regional de Saúde de LVT só foi elaborado dois anos depois, seguindo-se o da ARS do Norte e o dos Açores, e por fim, quatro anos depois, o da ARS Centro.

\section{OS PLANOS LOCAIS DE SAÚDE}

Apesar da descrição de quase todos os planos locais de saúde no documento de Redesenha dos Planos de Saúde $(D G S, 2015)^{\text {b }}$, com exceção do pertencente à ULS do Litoral Atentejano e do correspondente à ACES Oeste-Sul, realizada uma pesquisa online, pelo motor de busca Google, e usando a palavra-chave "plano local de saúde", dos 54 ACES e ULS existentes em Portugal Continental, só se teve acesso aos seguintes Planos Locais de Saúde:

1. Plano local de saúde 2014-2016, do ACES Amadora (2014);

2. Plano local de saúde 2015, do ACES Barcelos/Esposende (2015);

3. Plano local de saúde 2015-2017, do ACES Cascais (2015);

4. Plano local de saúde 2012-2016, do ACES Entre Douro e Vouga I, Feira/Arouca (2012);

5. Plano local de saúde 2011-2016, do ACES Entre Douro e Vouga II, Aveiro Norte (2011);

6. Plano local de saúde 2011-2016, do ACES Gaia e Espinho (2011);

7. Plano Local de saúde 2011-2016, do ACES Grande Porto IV Maia (2011);

8. Plano Local de saúde 2011-2016, do ACES Grande Porto VIII Gaia (2011);

9. Plano Local de Saúde 2014-2016, do ACES Lisboa Ocidental e Oeiras (2014);

10. Plano Local de Saúde 2014-2016, do ACES Oeste Norte (2014);

11. Plano Local de Saúde 2011-2016, do ACES Porto Oriental (2011);

12. Plano Local de Saúde 2015-2017, do ACES Sintra (2015);

13. Plano Local de Saúde 2011-2016, da Unidade Local de Saúde de Matosinhos (2011).

Não se encontraram dados nem Planos Locais de Saúde, por ilha, referentes às Regiões Autónomas.

Na Conferência "Plano Nacional de Saúde - Desafios Presentes", que decorreu no dia 19 de setembro deste ano, em Loures, foi apresentado o Plano Local de Saúde - Revisão e Extensão a 2020 do Agrupamento de Centros de Saúde Loures Odivelas (DGS, 2016)a , mas, mais uma vez, não se encontrou o documento online, mesmo usando as palavras "Plano Local de Saúde Loures", para pesquisa no Google. O mesmo se passou com o Plano Local de Saúde de Évora, apresentado a 28 de Setembro desde ano, na conferência "Pensar Saúde, Alinhar Estratégias", em Évora. 
A recolha de dados a nível local é a base da estratégia local de saúde em cada município, o principal instrumento para a realização dos grandes objetivos do Plano Nacional de Saúde, e uma peça fundamental para o sucesso da sua implementação em todo o território (Sakellarides, Gonçalves e Santos, 2010), pelo que é preciso agir para ultrapassar as graves lacunas da realidade encontrada.

Apesar da avaliação do papel dos municípios na implementação do Plano Local de Saúde (e logo, no Plano Nacional de Saúde) não fazer parte deste trabalho, há que salientar o trabalho desenvolvido pela Câmara Municipal de Cascais (2016), que publicou já a "Estratégia local de promoção da saúde 2016-2020" (enquadrada com o período de extensão do Plano Nacional de Saúde a 2020), assim como o trabalho da Câmara Municipal de Lisboa (2015), na realização do Plano de Desenvolvimento de Saúde e Qualidade de Vida da Cidade de Lisboa. Estes documentos e outros que estarão a ser preparados a nível dos municípios, por todo o território, são de extrema importância e deveriam estar também alinhados com os respetivos Planos Locais de Saúde, num trabalho a ser futuramente realizado entre ACES/ ULS e autarquias. Esta concertação de objetivos, metas, indicadores e estratégias prioritárias de ação a nível local, terá um papel determinante na implementação dos eixos estratégicos do Plano Nacional de Saúde e na preciosa recolha de dados a nível local, que permitirá a tomada de decisão informada por parte do Governo, quando se realizar a avaliação da implementação dos Planos Locais de Saúde (pelos ACES/ ULS), dos Planos Regionais de Saúde (pelas ARS e Secretarias Regionais de Saúde) e do Plano Nacional de Saúde (pelo Ministro).

OS PLANOS LOCAIS DE SAÚDE SÃO COERENTES COM O PLANO NACIONAL DE SAÚDE? QUAL A MELHOR FORMA DE IMPLEMENTAR O PLANO NACIONAL DE SAÚDE A NIVEL LOCAL?

Um estudo efetuado no âmbito do estágio relativo ao Internato Médico realiza a avaliação do grau de integração dos objetivos e dimensões dos eixos presentes no Plano Nacional de Saúde nos Planos Locais de Saúde, com base no documento de Redesenha dos Planos de Saúde $(D G S, 2016)^{c}$. Esta análise é importante porque permitirá avaliar quais as prioridades definidas a nível local, quais as estratégias locais mais prevalentes e indicadores que pretendem monitorizar, avaliando se estão de acordo com o Plano Nacional de Saúde.

Os resultados deste estudo indicam que há uma mediana integração dos eixos nos Planos Locais de Saúde, parcialmente justificada pelo facto de no período de cinco anos terem existido três Planos Nacionais de Saúde (DGS, 2016) ${ }^{c}$. O estudo concluiu que há necessidade de criar um manual com orientações para a realização dos Planos Locais de Saúde, de forma a melhor uniformizar a sua conceção (DGS, 2016) ${ }^{c}$, o que se considera fundamental para a implementação do PNS. 
Working Paper

Departamento de Ciências Económicas e Empresariais Universidade Autónoma de Lisboa

A própria DGS fez este ano uma análise interessante do grau de integração dos objetivos e dimensões dos eixos presentes no PNS 2012-2016 com Extensão a 2020, nos Planos Locais de Saúde (DGS, 2016) ${ }^{\mathrm{b}}$ e apresentou os resultados que se detalham na Tabela 1.

Tabela 1: Grau de integração dos objetivos e dimensões dos eixos presentes no PNS 20122016-2020, nos Planos Locais de Saúde

\begin{tabular}{|c|c|c|}
\hline \multicolumn{2}{|c|}{ PNS 2012-2016 com Extensão a 2020} & \multirow{2}{*}{$\begin{array}{c}\text { Número de ACES com referência ao tema } \\
\text { (\% num total de } 54 \text { ACES no Continente) } \\
\text { nos PLS ou respetivo esboço }\end{array}$} \\
\hline Eixos Estratégicos & Categorias & \\
\hline \multicolumn{2}{|l|}{ Cidadania em Saúde } & $42(77,7 \%)$ \\
\hline \multirow[t]{4}{*}{ Cidadania em saúde } & Literacia & $42(77,7 \%)$ \\
\hline & Participação & $4(7,4 \%)$ \\
\hline & Empowerment & $4(7,4 \%)$ \\
\hline & Informação e comunicação & $6(11,1 \%)$ \\
\hline \multicolumn{2}{|l|}{ Equidade e Acesso } & $31(57,4 \%)$ \\
\hline \multirow[t]{4}{*}{ Equidade e Acesso } & Acesso & $19(35,2 \%)$ \\
\hline & Desigualdades & $1(1,9 \%)$ \\
\hline & Necessidades & $2(3,7 \%)$ \\
\hline & Oferta de cuidados & $31(57,4 \%)$ \\
\hline \multicolumn{2}{|l|}{ Qualidade na Saúde } & $16(29,6 \%)$ \\
\hline \multirow[t]{4}{*}{ Qualidade na Saúde } & Patient Safety & $8(14,8 \%)$ \\
\hline & Satisfação & $3(5,6 \%)$ \\
\hline & Avaliação de intervenções & $6(11,1 \%)$ \\
\hline & Ganhos e melhorias & $4(7,4 \%)$ \\
\hline \multicolumn{2}{|l|}{ Políticas Saudáveis } & $38(70,4 \%)$ \\
\hline \multirow[t]{5}{*}{ Políticas Saudáveis } & Intersetorial & $38(70,4 \%)$ \\
\hline & Promover parcerias & $37(68,5 \%)$ \\
\hline & Investigação & $4(7,4 \%)$ \\
\hline & Visão & $2(2,7 \%)$ \\
\hline & Políticas saudáveis (geral) & $1(1,9 \%)$ \\
\hline \multicolumn{3}{|l|}{ Orientações } \\
\hline \multicolumn{2}{|c|}{ Prevenção e controlo da doença } & $50(92,6 \%)$ \\
\hline \multicolumn{2}{|c|}{ Promoção e proteção da saúde } & $50(92,6 \%)$ \\
\hline \multicolumn{2}{|c|}{ Colaboração intersectorial } & $38(70,4 \%)$ \\
\hline \multicolumn{2}{|l|}{ Capacitação } & $18(33,3 \%)$ \\
\hline \multicolumn{2}{|c|}{ Promoção de Ambientes Saudáveis } & $27(50 \%)$ \\
\hline \multicolumn{2}{|l|}{ Boas Práticas } & $29(53,7 \%)$ \\
\hline \multicolumn{2}{|l|}{ Saúde Global } & $0(0 \%)$ \\
\hline
\end{tabular}

Fonte: Adaptado de (DGS, 2016) ${ }^{\mathrm{b}}$ 
Apesar do estudo ter acedido apenas a doze Planos Locais de Saúde completos, os resultados apresentados indicam que há uma estrutura muito heterogénea dos Planos Locais de Saúde dos ACES, com pouca uniformização dos critérios utilizados para a sua conceção, embora a maioria estivesse ainda numa fase inicial da sua construção.

Por outro lado, há uma referência às categorias de cada um dos eixos estratégicos definidos no atual PNS muito menor que o espectável. O que se poderá interpretar do facto de apenas cerca de $30 \%$ dos ACES tenha, em 2016, incluído o eixo estratégico de Qualidade na Saúde no seu Plano Local de Saúde (ou Esboço do Plano)? E que apenas cerca de $60 \%$ se foque num dos dois principais eixos estratégicos da "Saúde 2020", relacionado com a saúde para todos e com a redução da iniquidade em saúde?

É muito urgente agir para que a política de saúde a nível central chegue às entidades prestadoras de cuidados a nível local e para que possa ser implementada em todo o território. Sugere-se que o Governo adote um procedimento mais eficaz para implementação do atual Plano Nacional de Saúde. Tenhamos como exemplo a adaptação do "Pacote de Implementação" da política europeia "Saúde 2020" (WHO, 2012), composto por vários passos, à realidade nacional:

1 Passo: Apresentar o Plano Nacional de Saúde a diferentes stakeholders

A Direção Geral de Saúde poderia considerar a apresentação do documento, aprovado pelo Gabinete do Ministro, à alta direção e à direção intermédia da ACSS, das ARS, do Instituto Português do Sangue e da Transplantação, do Instituto da Droga e Toxicodependência, do INFARMED, do INEM, do INSA, às universidades com formação na área saúde, às ordens dos profissionais de saúde, às associações de doentes e de profissionais, aos principais grupos de seguros, à Segurança Social, à Agência Portuguesa do Ambiente e à comunicação social.

Mais tarde, respeitando um período previamente estabelecido, o Plano Nacional de Saúde deveria ser apresentado às organizações de saúde em Portugal, divididas pelo território associado a cada ARS ou Secretaria Regional de Saúde. Este Plano deveria ser apresentado em conjunto com o respetivo Plano Regional de Saúde. O público-alvo seria a direção dos ACES e ULS, dos hospitais públicos, privados e do setor social, a direção técnica e proprietários das farmácias comunitárias, das clínicas médicas, dos laboratórios de análises clínicas, dos laboratórios da indústria farmacêutica, das IPSS, seguradoras, etc.. Nestas sessões de apresentação, deveriam estar também presentes as direções das escolas, das autarquias, dos ginásios e clubes desportivos, das prisões e os órgãos locais e regionais da comunicação social. Simultaneamente deveria ser publicamente assumido um prazo para a execução do Plano Local de Saúde, pelos ACES e ULS e deveria ser atribuída a responsabilidade pela sua publicação nos media locais e divulgação aos profissionais de saúde, por comunicação interna através das unidades de saúde onde colaboram.

2ํㅜ Passo: Realizar uma análise da situação nacional, regional e local.

Para a implementação do Plano Nacional de Saúde, é necessário que haja uma análise sistemática da situação do país. Esta análise sustentada de dados epidemiológicos de base 
populacional e institucional, a nível local, é fundamental para a concretização dos Perfis Locais de Saúde, para que se percebam quais os determinantes da doença, quais os fatores protetores e de risco da doença no território correspondente. Esta informação é importante não só para definir prioridades de ação em ambiente de escassos recursos, essenciais à construção dos planos de saúde a nível nacional, regional e local, como para realizar uma monitorização da evolução da saúde pública no país e avaliação da implementação das políticas públicas.

3 ํ Passo: Apostar fortemente na monitorização e controlo.

Para se proporcionar ao Governo informação atualizada sobre o estado da saúde da população a nível local, regional e nacional, é preciso que seja implementado um processo sistemático de recolha de dados atualizados, essencial à tomada de decisão informada. Só a compilação destes dados permitirá analisar a capacidade de implementação das políticas públicas de saúde a nível local (e nacional) e o impacto destas políticas na saúde da população.

Caso não haja esta recolha sistemática de dados, o controlo fica cego e incapacitado de agir perante a mudança e a realidade do quotidiano.

4ํ Passo: Implementar uma abordagem de priorização da saúde, transversal a todos os membros do governo e da sociedade, com atribuição de papéis e de responsabilidades aos diferentes atores.

É fundamental que haja saúde em todas as políticas públicas, independentemente do Ministério que as publique, e que todas tenham em consideração o bem-estar físico, mental e social da população. É também importante que a sociedade civil exerça pressão no sentido de se colocar a saúde da população à frente dos interesses económicos e comerciais. É por isso importante que o Governo incentive esta preocupação na sociedade, valorizando a saúde pública. É também fundamental que atribua responsabilidades a cada Português pela proteção da sua saúde, e que responsabilize a direção de cada membro que compõe o Serviço Nacional de Saúde, pelos resultados previstos alcançar, face aos recursos disponíveis e às necessidades populacionais a que deve dar resposta.

5o Passo: Dar relevância aos determinantes sociais de equidade em saúde, sexo e direitos humanos. Uma das grandes prioridades da política de saúde na Europa é a redução da iniquidade social. É preciso proporcionar recursos e condições semelhantes para pessoas/ episódios com características semelhantes. De acordo com a Constituição Portuguesa, no país todos têm o direito à saúde. Na realidade, temos um Serviço Nacional de Saúde que é universal (para todos), global (disponível em todo o território) e tendencialmente gratuito, tendo em conta as condições económicas e sociais dos cidadãos, uma vez que se tornou insustentável para o país suportar a crescente despesa com a saúde dos cidadãos apenas com financiamento público. $O$ acesso ao sistema não é igual, é equitativo. Isto quer dizer que há diferentes condições de acesso aos cuidados de saúde por razões relacionadas com diferentes estados de gravidade associados a diferentes patologias (com diferente letalidade, morbilidade, frequência de sintomas ou pela pertença a um grupo de risco, como as grávidas, crianças e idosos, etc.). Pode-se dizer que existem em Portugal condições iguais de acesso ao Serviço Nacional de Saúde, para 
Working Paper

Departamento de Ciências Económicas e Empresariais

Universidade Autónoma de Lisboa

pessoas em iguais situações, o que se considera vantajoso e justo para a população que é tratada no setor público. Se compararmos no entanto as condições de acesso ao setor público e ao setor privado (mesmo às instituições com contratos ou convenções com o serviço público) daqueles que não têm capacidade para pagar a parte que os sub-sistemas de saúde (ADSE, ADME, SAMS, etc.) não financiam, não há equidade, já que é normal que o acesso ao setor privado seja mais rápido. Podemos contudo afirmar que em Portugal não há diferentes condições de acesso por motivos relacionados com a raça, etnia ou religião, o que é um bom indicador da humanização do SNS, que não nega o atendimento aos sem-abrigo e a imigrantes ilegais.

60 Passo: Alinhamento da conduta das políticas de saúde a nível local, regional e nacional.

Os Planos Locais de Saúde e de estratégias locais de saúde devem ser desenvolvidas com base na estratégia regional (explícita no Plano Regional de Saúde), que, por sua vez, deve refletir as diretrizes de ação do Governo a nível nacional (que constam no Plano Nacional de Saúde). Apesar da linha de conduta política ser a mesma, há diferenças entre os diferentes níveis de Planos, atendendo às especificidades de cada local ou região e aos resultados dos dados epidemiológicos analisados, na parte do território correspondente. Só assim se garante a implementação do Plano Nacional de Saúde em todo o território e a sua adequação à realidade particular de cada zona.

$7^{\circ}$ Passo: Fortalecimento da saúde pública e da capacidade dos serviços de saúde.

Um dos pilares essenciais à implementação de um Plano Nacional de Saúde é a centralização do sistema de saúde nas pessoas. Como os recursos de cada organização de saúde são limitados, é preciso que haja uma cooperação estreita entre organizações da mesma região para se dar resposta às necessidades de saúde das populações, dos setores público, privado e social. É ainda essencial apostar num papel crescente dos cuidados primários na sociedade, com maior ação na promoção da saúde, na prevenção da doença e na proximidade com a população residente, bem conectados com os cuidados secundários, onde se deve apostar cada vez mais na especialidade das organizações por patologia e na complementaridade destes serviços por região, para maior eficiência e eficácia do sistema. A estreita relação entre os cuidados terciários e os secundários, permite também o aumento da esperança de vida da população, num grupo populacional muito fragilizado (idosos, politraumatizados, etc.).

8 Passo: Realizar um cronograma de implementação do Plano Local de Saúde.

É importante haver prazos bem definidos para a elaboração do Plano Regional de Saúde, depois de apresentado o Plano Nacional de Saúde, e conceção do Plano Local de Saúde, depois de publicado o Plano Regional. No entanto, para implementação do Plano Local de Saúde, é preciso listar os procedimentos a executar localmente e os objetivos a alcançar, e atribuir a cada um deles um prazo, que deve ser respeitado e monitorizado, com uma periodicidade adequada (avaliar cerca de 10 indicadores relacionados com as prioridades assumidas trimestralmente e os restantes pelo menos uma vez por ano) para o alcance das metas do Plano Local de Saúde. Caso se verifique um desvio às metas, é preciso agir em conformidade, avaliando o motivo e 
Working Paper

Departamento de Ciências Económicas e Empresariais

Universidade Autónoma de Lisboa

agindo sobre ele de imediato. Se esta monitorização intermédia não for realizada, perde-se a oportunidade de agir atempadamente sobre algum desvio negativo. O governo devia ter acesso ao relatório anual de implementação dos Planos por ACES/ ULS e por ARS/ Secretaria Regional de Saúde. Se as metas dos Planos Locais de Saúde forem alcançadas, também o serão as dos Planos Regionais de Saúde correspondentes, levando ao alcance das metas nacionais do Plano Nacional de Saúde. Caso isto não aconteça, dificilmente as metas nacionais serão alcançadas e os compromissos internacionais do Governo cumpridos.

De notar que, até ao final do ano corrente, as metas e indicadores dos Planos Locais de Saúde estão em fase de avaliação, não se encontrando para já, dados disponíveis sobre o alcance das metas estabelecidas para 2016 a nível local, nem a nível nacional, que nos possam elucidar sobre a implementação das estratégias de ação definidas pelo Governo.

\section{OPINIÃO DOS PROFISSIONAIS DE SAÚDE EM ATIVIDADE}

Constatou-se haver entre os profissionais de saúde um desconhecimento generalizado sobre as metas e eixos prioritários dos Planos Locais e Nacional de Saúde, revelando a necessidade de maior comunicação e proximidade entre o Ministério da Saúde, os profissionais de Saúde e a população, para que todos sejam motivados para trabalhar para um objetivo comum: o ganho em saúde dos portugueses.

\section{CONCLUSÃO}

Conclui-se assim que a implementação dos Planos Locais de Saúde, documento base da política de saúde nacional a nível local, se encontra ainda numa fase muito precoce e que os Planos que existem não refletem os eixos estratégicos do Plano Nacional de Saúde 2012-2016-2020 da forma ambicionada, sugerindo que é preciso atuar rapidamente para resolução desta situação. Sugere-se ainda que as Secretarias Regionais de Saúde das Regiões Autónomas implementem as estratégias locais de saúde por ilha, atendendo à grande heterogeneidade populacional e de recursos entre elas.

\section{REFERÊNCIAS BIBLIOGRÁFICAS}

ACES Amadora (2014). Plano local de saúde 2014-2016. Amadora: ARS LVT. Recuperado em 28 de Abril de 2016, de http://www.cmamadora.pt/images/artigos/saudavel/noticias/ pdf/2014/plano_local_saude.

ACES Barcelos/Esposende (2015). Plano local de saúde 2015. Barcelos: ARS Norte. Recuperado em 28 de Abril de 2016, de http://portal.arsnorte.min-saude.pt/portal/page /portal/ARSNorte/Sa\%C3\%BAde\%20P\%C3\%BAblica/Planeamento\%20em\%20Sa\%C3\%BA de/Perfis\%20de\%20Sa\%C3\%BAde/PLS/70767DD3042C02D9E040140A11026757. 
ACES Cascais (2015). Plano local de saúde 2015-2017. Cascais: ARS LVT. Recuperado em 9 de Outubro de 2016, de http://www.cm-cascais.pt/sites/default/files/anexos/gerais/new/ cascais_plano_local_de_saude_2015-2

ACES Entre Douro e Vouga I, Feira-Arouca (2012). Plano local de saúde 2012-2016. Aveiro: ARS Norte. Recuperado em 16 de Outubro de 2016, de http://rede-social.cm-feira.pt/redesocial/instrumentos-de-diagnostico-e-planeamento/documentos/ples-aces-feira-arouca-2012 $-2016$

ACES Entre Douro e Vouga II, Aveiro Norte (2011). Plano local de saúde 2011-2016. Aveiro: ARS Norte. Recuperado em 28 de Abril de 2016, de http://www.docvadis.pt/usf-vale-dovouga/document/usf-vale-do-vouga/plano_local_de_saude_aces_aveiro_norte_2011_2016 /fr/metadata/files/0/file/Plano\%20Local\%20de\%20Sa\%C3\%BAde\%20\%20Aveiro\%20Norte.

ACES Gaia e Espinho (2011). Plano local de saúde 2011-2016. Gaia: ARS Norte. Recuperado em 28 de Abril de 2016, de http://portal.arsnorte.min-saude.pt/portal/page/portal/ ARSNorte/Sa\%C3\%BAde\%20P\%C3\%BAblica/Planeamento\%20em\%20Sa\%C3\%BAde/Perf is\%20de\%20Sa\%C3\%BAde/PLS/70CB6ED0337C63E7E040140A11027471.

ACES Grande Porto IV Maia (2011). Plano Local de saúde 2011-2016. Maia: ARS Norte. Recuperado em 28 de Abril 2016, de http://portal.arsnorte.min-saude.pt/portal/page/ portal/ARSNorte/Conte\%C3\%BAdos/Documentos/ACES_Maia_PLS_2011_2016.

ACES Grande Porto VIII Gaia (2011). Plano Local de saúde 2011-2016. Gaia: ARS Norte. Recuperado em 28 de Abril de 2016, de https://www.google.pt/?ion=1\&espv= 2\#q=ACES+Grande+Porto+ VIII+Gaia.

ACES Lisboa Ocidental e Oeiras (2014). Plano Local de Saúde 2014-2016. Lisboa: ARS LVT. Recuperado em 9 de Outubro de 2016, de http://www.arslvt.minsaude.pt/uploads/writer_file/document/1034/Plano_Local_Sa_de_v4-_Vers_o_Final.pdf

ACES Oeste Norte (2014). Plano Local de Saúde 2014-2016. Caldas da Rainha: ARS LVT. Recuperado em 28 de Abril de 2016, de http://www.arslvt.min-saude.pt/uploads/ writer_file/document/1034/Plano_Local_Sa_de_v4-_Vers_o_Final.

ACES Porto Oriental (2011). Plano Local de Saúde 2011-2016. Porto: ARS Norte. Recuperado em 28 de Abril de 2016, de http://portal.arsnorte.min-saude.pt/portal/page/ portal/ARSNorte/Sa\%C3\%BAde\%20P\%C3\%BAblica/Planeamento\%20em\%20Sa\%C3\%BA de/Perfis\%20de\%20Sa\%C3\%BAde/PLS/ACES\%20Porto\%20(Ocidental\%20e\%20Oriental).

ACES SINTRA (2015). Plano Local de Saúde 2015-2017. Sintra: ARS LVT. Recuperado em 28 de Abril de 2016, de http://www.arslvt.min-saude.pt/uploads/writer_file/document /2192/Plano_Local_de_Saude_de_Sintra.

Administração Regional de Saúde do Centro (2015). Plano Regional de Saúde do Centro 20152016. Coimbra: ARS Centro. Recuperado em 28 de Abril de 2016, de http://www.arscentro.min-saude.pt/Institucional/Documents/monitoriza\%C3\%A7\%C3\%A30\% 20e\%20avalia\%C3\%A7\%C3\%A3o/Plano\%20Regional\%20Saude\%20ARS\%20Centro\%202 015-2016. 
Administração Regional de Saúde de Lisboa e Vale do Tejo (2013). Plano Regional de Saúde 2013-2016. Lisboa: ARS LVT. Recuperado em 9 de Outubro de 2016, de http://www.arslvt.min-saude.pt/uploads/writer_file/document/287/ARSLVT_-_PLANO_ REGIONAL_DE_SA_DE_2013-2016_rev._09_junho_.pdf

Administração Regional de Saúde do Norte (2014). Plano Regional de Saúde do Norte 20142016. Porto: ARS Norte. Recuperado em 28 de Abril de 2016, de http://portal.arsnorte.minsaude.pt/portal/page/portal/ARSNorte/Conte\%C3\%BAdos/Sa\%C3\%BAde\%20P\%C3\%BAbli ca\%20Conteudos/PlanoRegionalSaudeNorte_2014_2016.

Câmara Municipal de Cascais (2016). Cascais: Estratégia local de promoção da saúde 20162020. Cascais: CMC. Recuperado em 9 de Outubro de 2016, de http://www.sermais.pt/media /86/File/Cascais_EstrategiaLocalDePromocaoDaSaude2016_2020.pdf

Câmara Municipal de Lisboa (2015). Plano de Desenvolvimento de Saúde e Qualidade de Vida da Cidade de Lisboa: Volume I- Perfil Municipal de Saúde de Lisboa. Lisboa: CML. Recuperado a 9 de Outubro de 2016, de http://isboasolidaria.cm-lisboa.pt/documentos/ 1443532683B5fMF6 um6Ap 74HT0.pdf

DGS (2015)a. Plano Nacional de Saúde 2012-2016: Revisão e Extensão a 2020. Lisboa: DGS. Recuperado em 28 de Abril de 2016, de http://pns.dgs.pt/pns-revisao-e-extensao-a-2020/.

DGS (2015) . Redesenha dos Planos de Saúde: Nacional-Regionais-Locais, Plano Nacional de Saúde e Estratégias Locais de Saúde. Lisboa: DGS. Recuperado em 9 de Outubro de 2016, de http://1nj5ms2lli5hdggbe3mm7ms5.wpengine.netdna-cdn.com/files/2015/12/Resenha PlanosSa\%C3\%BAdeNacionalRegionaisLocais1.pdf.

DGS (2016)a . Os desafios presentes do Plano Nacional de Saúde. Lisboa: DGS. Recuperado em 5 de Outubro de 2016, de https://www.dgs.pt/em-destaque/os-desafios-presente-doplano-nacional-de-saude-.aspx.

DGS (2016) ${ }^{\text {b }}$ Análise dos Planos Locais de Saúde. Lisboa: DGS. Recuperado em 9 Outubro de 2016, de https://www.dgs.pt/em-destaque/integracao-dos-planos-locais-de-saude-com-oplano-nacional-de-saude-.pdf

DGS (2016)c. Integração dos Planos Locais de Saúde com o Plano Nacional de Saúde. Lisboa: DGS. Recuperado em 9 Outubro de 2016, de https://www.dgs.pt/em-destaque/integracaodos-planos-locais-de-saude-com-o-plano-nacional-de-saude-.aspx

Secretaria Regional de Saúde da Região Autónoma dos Açores (2014). Plano Regional de Saúde 2014-2016. Angra do Heroísmo: SRSRAA. Recuperado em 28 de Abril de 2016, de https://www.azores.gov.pt/NR/rdonlyres/C1434B21-D0C0-4B26-9A3B17254892AD0F/0/PRS 20142016.

Secretaria Regional dos Assuntos Sociais da Região Autónoma da Madeira (2011). Plano Estratégico do Sistema Regional de Saúde: 2011-2016. Funchal: SRASRAM. Recuperado em 28 de Abril de 2016, de http://iasaude.sras.gov-madeira.pt/documentos/WEB/Anexos/plano1.

Sakellarides, C., Gonçalves, C. e Santos, A. (2010). Plano Nacional de Saúde e as Estratégias Locais de Saúde. Lisboa: ENSP. https://www.google.pt/\#q=SAKELLARIDES\%2C+C 
.\%2C+GON\%C3\%87ALVES\%2C+C.+E+SANTOS\%2C+A.+(2010).+Plano+Nacional+de+Sa $\%$ C3\%BAde+e+as+Estrat\%C3\%A9gias+Locais+de+Sa\%C3\%BAde.

Unidade Local de Saúde de Matosinhos (2011). Plano Local de Saúde 2011-2016. Matosinhos: ARS Norte. Recuperado em 16 de Outubro de 2016, de http://www.ulsm.minsaude.pt/ResourcesUser/Unidade_de_Saude_Publica/Plano_Local_Saude_ULSM_2011_20 16.pdf.

World Health Organization - Regional Office for Europe (2012). Health 2020: the European policy for health and well-being Implementation package. Recuperado a 10 de Outubro de 2016, de http://www.euro.who.int/en/health-topics/health-policy/health-2020-the-european-policy-forhealth-and-well-being/implementation-package

WHO Europe (2015). Interim Report on the National Health Plan: Revision and Extension to 2020. Recuperado a 11 de Outubro de 2016, de http://pns.dgs.pt/files/2015/07/2015_OMS_InterimReport-on-the-National-Health-Plan_revision-and-extension-to.pdf 